\title{
Contribution à l'étude de la dilution des effluents radio-actifs dans le Rhône par le rejet expérimental de rhodamine $B$
}

\author{
A contribution to the study \\ of radio-active waste dilution in the Rhone \\ involving tests with a rhodamine $B$ tracer
}

\author{
PAR J. RODIER ET M. MARICHAL \\ SEIVICE DE PROTECTION CONTRE LES RADIATIONS, CENTRE DE MARCOULE
}

\begin{abstract}
L'emploi de la rhodamine comme traceur chimique a permis de suivre l'évolution du mélange des effluents du Centre de Marcoule aux eaux du Rhône. La dispersion est déjà satisfaisante d̀ moins de $4 \mathrm{~km}$ en aval de la conduite des rejets, et l'homogénéité peut être considérée comme atteinte au pont de Roquemaure et parfaite à Avignon. Cette étude a montré que les veines préférentielles où se concentrerait l'écoulement des effuents radio-actifs n'existent pas. Elle a permis de préciser en outre les emplacements les plus représentatifs des points d'échantillonnage des eaux du Rhône au cours des rejets.
\end{abstract}

\begin{abstract}
The process whereby waste from the Marconle plant mixes with the water in the Rhone was followed in tests with rhodamine as a chemical tracer. Satisfactory dispersion was noted less than $4 \mathrm{~km}$ downstream from the waste discharge duct outlet, and the degree of homogeneity was considered to be satisfactory at the bridge of Roquemaure, and perfect at Avignon. This investigation not only revealed a complete absence of any preferential flow paths containing high radio-active waste concentrations, but it also enabled the most representaive points to be selected at which to take Rhone water samples during future radio-active waste discharges.
\end{abstract}

Les eaux résiduaires radioactives des diverses installations du Centre de Marcoule sont contaminées à divers degrés par des produits de fission et du plutonium. Leur rejet dans le Rhône ne peut se faire qu'après une épuration aussi complète que possible, pour éviter la pollution radioactive des eaux du fleuve.

Une station de traitement des effluents collecte toutes les eaux usées radioactives ou suspectes du Centre et les épure par des procédés physiques ou chimiques selon l'importance, la nature ou l'origine de la contamination. En même lemps, elles sont progressivement diluées, puis évacuées. Les traitements d'épuration, les dilutions et l'homogénéisation avec les eaux du
Rhône sont les trois facteurs qui tendent à maintenir le niveau de la radioactivité du fleuve à une valeur compatible avec la santé publique.

Le traitement appliqué aux effluents est lié au degré d'activité. Les effluents les plus actifs sont soumis à une précipitation de ferrocyanure de nickel en milieu neutre, suivie d'une précipitation de carbonate de chaux en milieu alcalin. Les effluents moyennement actifs ne subissent que la précipitation de carbonate de chaux en milieu alcalin. Dans les deux cas, les boues de floculation, qui retiennent la plus grande partie de la radioactivité sont stockées sous forme de déchets radioactifs solides. Une filtration sur diatomées complète le traitement des 
effluents à haute et moyenne activité. Les liquides clairs sont entreposés dans des bassins avant leur rejet au Rhône.

En cours d'épuration, les effluents sont dilués une première fois par l'adjonction des réactifs précipitants et d'une quantité variable d'eau. Le mélange est assuré par le brassage mécanique dans les appareils de traitement et par recyclage en circuit fermé dans les bassins.

Une seconde dilution se produit dans la conduite de rejet qui débouche dans la canalisation d'évacuation des eaux de refroidissement de la centrale électrique de la pile G 1 . Cette canalisation débite $3600 \mathrm{~m}^{3} / \mathrm{h}$; comme les bassins traités sont vidés à raison de $50 \mathrm{~m}^{3} / \mathrm{h}$ maximum, cette dilution est donc de 72 , au moins.

Une troisième dilution, la plus importante, se produit enfin dans le Rhône, dont le débit horaire moyen est de 5 à 6 millions de $m^{3}$. L'ordre de grandeur de la dilution totale est de 100.000 .

La conduite évacuant les effluents traités et dilués se termine par un tuyau de $50 \mathrm{~m}$ de long et de $900 \mathrm{~mm}$ de diamètre, percé latéralement de 10 tubulures et disposé sur le fond du lit du fleuve, dans le sens du courant, non loin de la rive droite. Le dessin des tubulures, leur orientation et l'emplacement de la conduite ont été particulièrement étudiés pour assurer un mélange aussi rapide et homogène que possible.

Le Service de Protection contre les Radiations du Centre de Marcoule contrôle non seulement la radioactivité de chaque bassin, avant son rejet, par des mesures et des analyses radiochimiques appropriées, mais aussi celle des eaux du Rhône, au cours de chaque rejet. Ainsi, huit échantillons sont prélevés en bateau, au moyen d'une pompe, dont la crépine d'aspiration plonge à $1 \mathrm{~m}$ sous l'eau, en des points choisis en fonction de l'hydrologie du fleuve. Ces points sont donnés dans la figure 1 et portent les numéros R.1 à R.8. Les huit prélèvements d'eau sont l'objet d'une analyse radiochimique délaillée, dont le but est de surveiller à la fois le niveau de la radioactivité du Rhône et la dispersion homogène des rejets au sein du fleuve.

Jusqu'à présent, l'ensemble des résultats de ces analyses n'a pas fourni la possibilité de situer avec exactitude l'endroit où le mélange du Rhône et des rejets pouvait être considéré comme homogène. Ceci tient surtout au fait que les mesures de radioactivité, en raison de l'importante dilution que subissent les effluents, sont presque toujours effectuées à la limite de la sensibilité des appareils. Pour préciser ce point important, une autre méthode a été recherchée pour matérialiser cette homogénéisation et préciser à quel endroit elle pouvait être considérée comme satisfaisante. C'est pourquoi un rejet d'une solution de rhodamine $\mathrm{B}$ a été réalisé dans les mêmes conditions qu'un rejet d'efluents radioactifs.

\section{Intérêt de la rhodamine comme traceur}

La dispersion d'une solution dans un volume d'eau aussi important que celui du Rhône peut s'étudier à l'aide de substances facilement déce. lables à doses très faibles. Dans ce genre d'expérimentation, le chlorure de sodium, le bichromate de potassium ou de sodium, la fluorescéine, la rhodamine, les traceurs radioactifs... sont les plus fréquemment utilisés.

Pour notre cas, le chlorure de sodium n'a pas été retenu en raison des énormes variations de la teneur en sodium des eaux du Rhône. In poids considérable de sel eut été nécessaire, pour pouvoir négliger les variations naturelles.

En solutions diluées, les bichromates ont l'inconvénient d'être instables en présence do matières organiques. Il en est de même pour la fluorescéine, qui est complètement décomposce en solution à 1 millionième au bout de 12 heures d'exposition à la lumière.

Les traceurs radioactifs, employés avec des activités faibles, s'étant révélés imprécis, notre choix s'est fixé sur la rhodamine $\mathbf{B}$, dont les propriétés conviennent particulic̀rement bien à l'étude envisagée.

La rhodamine $B$ est un colorant rouge du groupe des phtaléines répondant à la formule $\mathrm{C}_{28} \mathrm{H}_{31} \mathrm{O}_{3} \mathrm{~N}_{2} \mathrm{Cl}$, ou

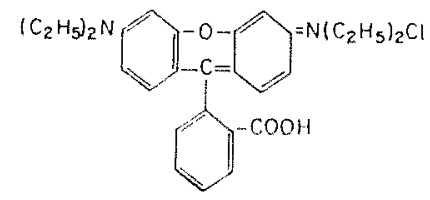

Dans un milieu naturel, et en solution diluce, elle reste stable pendant plus de deux semaines. L'intensité de la fluorescence n'est pas modifiée dans une gamme de $\mathrm{pH}$ comprise entre 4,0 et 10,5 . Enfin, la température n'influe pratiquement pas entre 12 et $28^{\circ} \mathrm{C}$.

La rhodamine $B$ est lrès peu toxique: des poissons survivent après avoir séjourné deux mois dans une solution à 100 millionièmes $\left(10^{-4}\right)$.

Son dosage se fait par photométrie de la fluorescence en ultraviolet, selon une méthode mise au point au laboratoire et décrite dans une note séparée $\left({ }^{*}\right)$. Cette technique très sensible permet d'apprécier des concentrations de $10^{-12}$, soit un millionième de milligramme de colorant par litre d'eau, mais n'est véritablement quanti-

( ) M. Mahlchal et R. BenorT: "Une méthode de dosage de la rhodamine $B$ dans les eaux waturelles". Service de Protection contre les Radiations du Centre de Marcoule, 1960. 
tative que pour des concentrations de l'ordre de $10^{-10}$, soit un dix-millième de milligramme par litre.

La rhodamine B est d'un emploi commode, car elle est soluble dans l'eau à raison de $2 \%$ en poids. Ses multiples usages comme colorant ou comme réactif analytique font qu'on la trouve facilement dans le commerce. Enfin, les très faibles concentrations nécessaires n'entraînent absolument aucune coloration des eaux, évitant ainsi toute difficulté avec les riverains.

\section{Conditions du rejet de rhodamine}

Le rejet de rhodamine s'est composé de $50 \mathrm{~kg}$ de colorant dissous dans $500 \mathrm{~m}^{3}$ d'eau. La solution a été préparée à l'avance dans un bassin muni d'un agitateur, puis a été rejetée à raison de $50 \mathrm{~m}^{3} / \mathrm{h}$ environ par la conduite de rejet $\mathrm{du}$ Centre de Marcoule. Le rejet a débuté le jeudi 2 juin 1960 à 5 heures du matin et a duré une dizaine d'heures, soit jusqu'à 15 heures. Dans ces conditions, la matinée a été consacrée aux prélèvements les plus voisins du site, jusqu'au pont de Roquemaure, et l'après-midi réservé aux prélèvements d'Avignon. Rappelons qu'il est admis qu^ le régime de l'écoulement des effluents dans le Rhône est atteint 4 heures après le début d'un rejet et qu'il faut environ 4 heures aux eaux du Rhône pour parcourir les $28 \mathrm{~km}$ qui séparent Marcoule d'Avignon, à la vitesse d'environ $7 \mathrm{~km} / \mathrm{h}$.

La quantité de rhodamine employée a été calculée sur la base d'un débit du Rhône de l'ordre de $1000 \mathrm{~m}^{3} / \mathrm{s}$, ce qui fail en 10 heures : $3,6.10^{\top} \mathrm{m}^{3}$. En se fixant une concentration finale de $10^{--9}$, ou 1 millième de milligramme par litre, soit dix fois la concentration pratiquement mesurable dans les conditions naturelles, on trouve une quantité totale théorique de $36 \mathrm{~kg}$. Les $14 \mathrm{~kg}$ supplémentaires ont été dissous, de manière à se trouver très largement dans la gamme de sensibilité de la méthode de dosage.

\section{Prélèvement \\ des échantillons d'eau du Rhône}

Plusieurs séries d'échantillons ont été prélevées en vue de déterminer leur teneur en rhodamine.

$1^{\circ}$ Trois sections ou tranches du Rhône ont été étudiées aux points suivants (fig. 1) :

a) P.K. 211, à hauteur de la Station de contrôle de la radioactivité des eaux de l'île de Codolet; 9 échantillons ont été pris en travers du fleuve, à $20 \mathrm{~m}$ environ l'un de l'autre, au moyen d'une pompe montée sur un bateau, et dont la crépine d'aspiration plonge à $1 \mathrm{~m}$ sous la surface.

b) P.K. 214, à hauteur de la Station de contrôle de la radioactivité des eaux de l'Ardoise; 8 échantillons ont été prélevés dans les mêmes conditions.

c) P.K 221,9, à hauteur de l'ancien bac de Roquemaure; 6 échantillons ont été recueillis de la même manière.

$2^{\circ}$ Une quatrième section a été étudiée au bac du pont Saint-Bénézet à Avignon. Le dispositif de pompage, spécialement conçu pour cet essai, permet de prélever des échantillons aux trois profondeurs de 1,2 ou $3 \mathrm{~m}$ sous la surface de l'eau; 8 échantillons ont été recueillis à $1 \mathrm{~m}$ de la surface, 6 à $2 \mathrm{~m}$ et 3 à $3 \mathrm{~m}$. En outre, à chaque rive, 1 échantillon a été prélevé à fleur d'eau.

$3^{\circ}$ En même temps, un bateau équipé d'une jompe plongeant à $1 \mathrm{~m}$ sous l'eau a effectué les prélèvements dans les mêmes conditions que



FIG. 1

Emplacement des points de prélèvements des eaux du Rhône. 
ceux qui sont habituellement faits au cours des rejets radioactifs. Pendant la prise d'échantillon, le bateau est maintenu à l'arrêt, dans le fil du courant, et sensiblement dans l'axe du chenal de navigation. Les prélèvements se font conventionnellement en remontant le fleuve, à partir du point le plus bas (P.K. 221). Les points de prélèvement sont situés comme suit (fig. 1) :
R.1 P.K. 208
Témoin amont;
R.2 P.K. 209
Témoin amont;

R.3 P.K. 210,550

$100 \mathrm{~m}$ en aval de la conduite de rejet;

R.4 P.K. 211

$550 \mathrm{~m}$ en aval de la conduite de rejet, à hauteur de la Station de l'île de Codolet;

R.5 P.K. 211,6 $1150 \mathrm{~m}$ en aval de la conduite de rejet, à hauteur de l'embouchure de l'Aygues;

R.6 P.K. 213,1 A hauteur de I'embouchure de la Cèze;

R.7 P.K. 214 A hauteur de la Station de l'Ardoise;

R.8 P.K. $221 \quad 10,5 \mathrm{~km}$ en aval de la condiuite de rejet, à hauteur du château de Montfaucon.
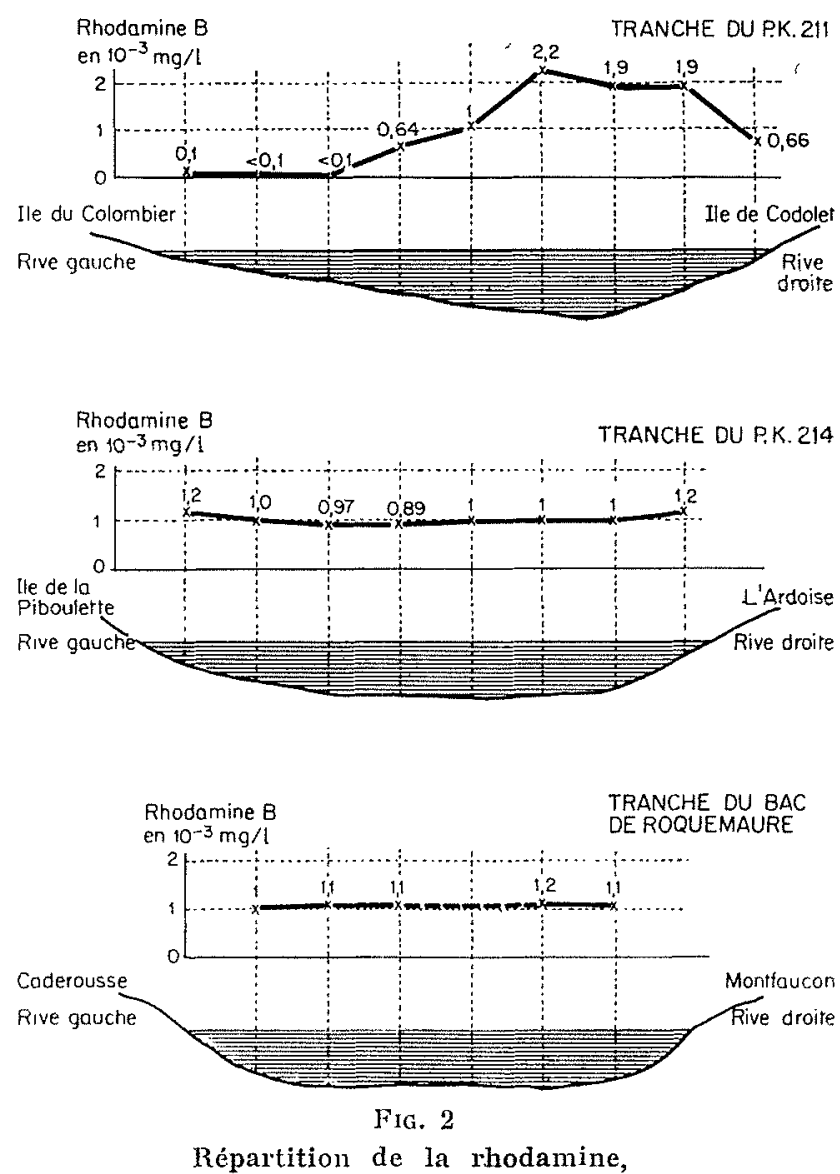

dans trois scctions transversales du Rhône.
Ces prélèvements à point fixe de la série R.1-R.8 ont été faits en double: une série le matin, une série l'après-midi.

Il a été procédé également à des échantillonnages en continu, selon une technique actuellement à l'étude. 100 litres d'eau sont pompés, pendant la marche du bateau et sur une distance de $1 \mathrm{~km}$, dans un fût d'où l'on soutire ensuite la quantité nécessaire aux analyses. Cette méthode a été imaginée pour essaȳer d'obtenir des échantillons plus représentatifs des eaux du Rhône, mais ne paraît pas apporter les améliorations espérées, comme nous le verrons plus loin. L'emplacement des lieux do prélèvement de cette série se répartit comme suit le long du Rhône (figs. 1):

E.1 entre les P.K. 208 et 209 (lémoin amont);

E.2 entre les P.K. 210,5 et 211,5 ;

E.3 entre les P.K. 212 et 213;

E.4 entre les P.K. 213 et 214;

E.5 entre les P.K. 214 et 215;

E.6 entre les P.K. 217 et 218;

E.7 entre les P.K. 220 et 221.

Les échantillons de cette série ont été récoltés dans la matinée, en même temps que ceux de la série R.1-R.8.

$4^{\circ}$ Des échantillons ont été puisés à plusieurs reprises ( $11 \mathrm{~h} 30,13 \mathrm{~h} 30$ et $15 \mathrm{~h} 30$ ) dans la cuve de la station de contrôle de la radioactivité des eaux du pont de Roquemaure, récemment équipée pour la mesure en continu de l'activité du Rhône. Cette station comprend essentiellement une pompe immergée fonctionnant en permanence et amenant l'eau dans une cuve oì plonge le cristal détecteur. L'intérêt de connaître les concentrations en rhodamine à cel endroit est évident.

5" Enfin, deux échantillons ont été également pris dans le bassin de la station des effluents contenant la solution de rhodamine, l'un vers 9 heures du matin, l'autre vers 15 heures.

\section{Etude des résultats des dosages de rhodamine}

Les dosages de rhodamine ont commencé immédiatement après les prélèvements, selon la méthode citée plus haut.

Les valeurs trouvées sont reportées dans les tableaux suivants et dans les figures 2,3 et 4 . Elles sont exprimées en millièmes de milligramme de colorant par litre d'eau.

Les concentrations trouvées dans les échantillons provenant $d u$ bassin contenant la solution à rejeter sont respectivement de $0,11 \mathrm{~g} /$ litre à 9 heures et de $0,12 \mathrm{~g} /$ litre à 15 heures. Elles 

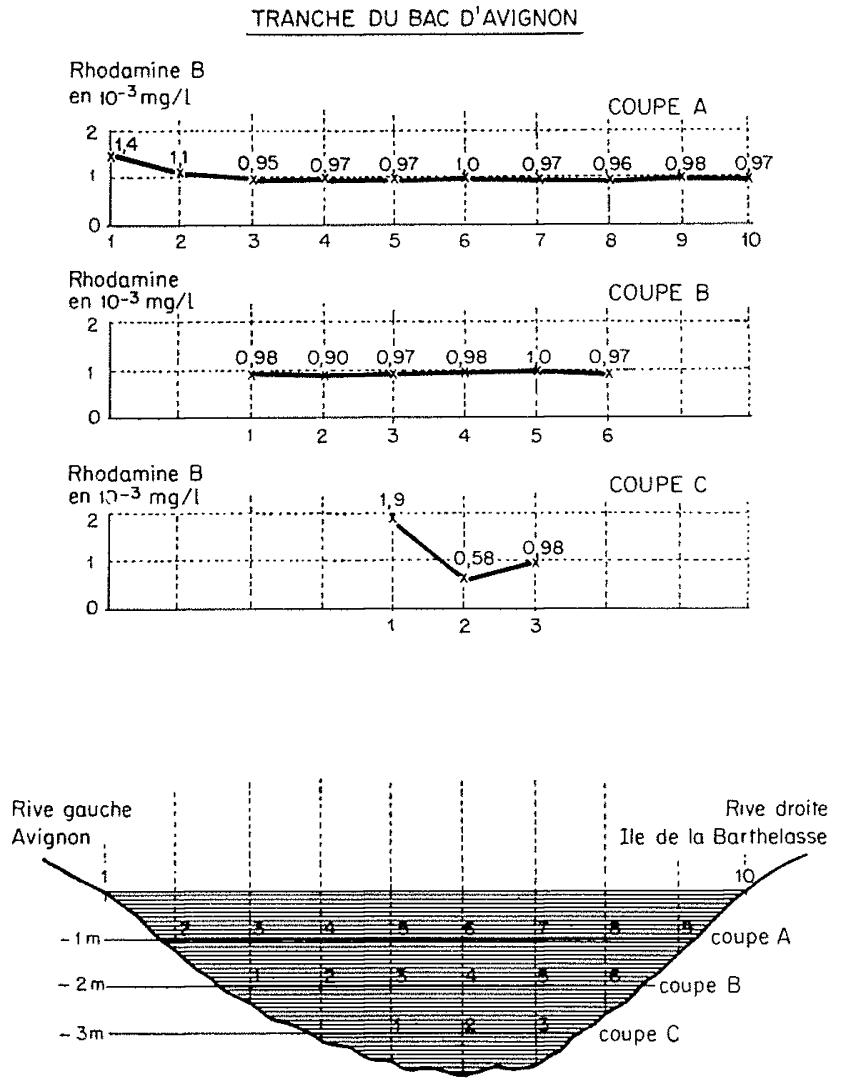

FIG. 3

Répartition de la rhodamine, dans une section transversale du Rhône, à différentes profondeurs.

montrent que cette solution était bien homogène et que les $50 \mathrm{~kg}$ de colorant s'étaient convenablement dissous dans $450 \mathrm{~m}^{3}$, lesquels ont été rejetés à raison de $40 \mathrm{~m}^{3} /$ heure environ.

Le débit moyen du Rhòne au cours des 10 heures de rejet a été de $1200 \mathrm{~m}^{3} / \mathrm{s}\left(1275 \mathrm{~m}^{3} / \mathrm{s}\right.$ à 7 heures et $1144 \mathrm{~m}^{3} / \mathrm{s}$ à midi, selon les données de l'échelle de Saint-Etienne-des-Sorts). La quantité totale d'eau dans laquelle se sont dispersés les $50 \mathrm{~kg}$ de rhodamine est donc de $43200000 \mathrm{~m}^{3}$, ce qui correspond théoriquement à une concentration de 1,16 millième de milligramme de rhodamine par litre d'eau. Cette valeur calculée correspond très bien avec les valeurs mesurées, surtout si l'on tient compte non seulement des volumes en présence, mais aussi de l'énorme différence entre le débit du rejet $\left(40 \mathrm{~m}^{3} / \mathrm{h}\right)$ et celui du Rhône (4 $\left.320000 \mathrm{~m}^{3} / \mathrm{h}\right)$.

Les valeurs observées dans la tranche du P.K. 211, la plus proche du point de rejet, montrent que, malgré la zone particulièrement turbulente qui entoure l'extrémité de la conduite, la dilution totale n'est pas encore atteinte. Au P.K. 211, le Rhône est relativement calme et les eaux voisines de la rive droite sont les plus chargées en colorant.

Au P.K. 214, à l'Ardoise, la dilution est beaucoup mieux répartie et peut déjà être considérée comme satisfaisante, grâce aux violents remous qui prennent naissance à l'embouchure de la Cèze. Quatre kilomètres à peine séparent la conduite de rejet de ce point.

Au bac de Roquemaure, situé à quelque $10 \mathrm{~km}$ en aval de la conduite, on retrouve également cette homogénéité. Les valeurs trouvées dans les échantillons prélevés dans la cuve de la station de contrôle de la radioactivité des eaux sont en accord avec ces résultats et correspondent remarquablement bien aux valeurs théoriques.

Les prélèvements d'Avignon font apparaître une excellente homogénéité sur toute la section du fleuve, quelle que soit la profondeur des pompages.

Les prélèvements en continu de la sério E.1-E.7, plus délicats à effectuer, du point de vue navigation, ne semblent pas apporter d'éléments supplémentaires dans l'appréciation de la contamination radioactive des eaux du Rhône. Ils confirment que la dilution est atteinte aux endroits mis en évidence par les prélèvements fixes de la série R.1-R.8.
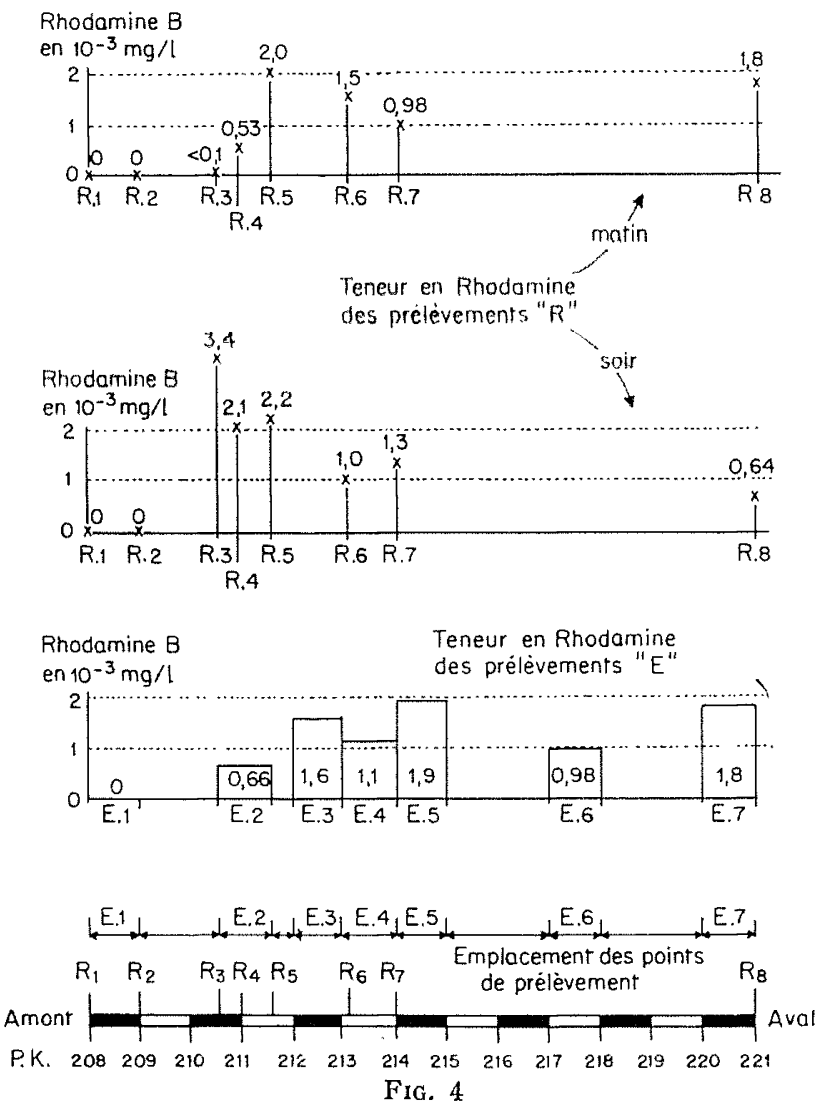

Répartition de la rhodamine

dans les prélèvements effectués au Rhône, au cours des rejets radioactifs du Centre de Marcoule. 


\section{Conclusions}

Ce rejet expérimental de rhodamine $B$, conduit exactement dans les mêmes conditions que les rejets d'effluents radioactifs du Centre de Marcoule, a fourni un certain nombre de renseignements intéressants.

- La concordance entre les concentrations calculées et les valeurs mesurées montre l'intérêt de la rhodamine et de son emploi comme traceur dans l'étude de phénomènes hydrologiques s'appliquant à des eaux aussi abondantes que celles du Rhône. Ce colorant et son mode de dosage peuvent donc s'utiliser d'une manière très générale, quel que soit le cours d'eau, ou même en mer.

- L'étude des résultats des dosages de rhodamine dans les diverses tranches du Rhône faisant l'objet de l'expérience, montre que la dispersion du rejet dans le courant s'effectue assez rapidement. En effet, dès le P.K. 211 (île de Codolet), le chenal de navigation est assez uniforme. Seule la partie gauche du fleuve n'est pas encore polluée par le rejet. Au P.K. 214, à l'Ardoise, on peut considérer la dilution comme satisfaisante; l'homogénéité du mélange peut être considérée comme atteinte à Roquemaure et parfaite à Avignon. L'ensemble des mesures ne fait pas apparaître l'existence de veines préférentielles où se concentrerait l'écoulement des effluents.

- En ce qui concerne le contrôle des rejets radioactifs, la multiplication des prélèvements se révèle inutile, d'autant que certains points d'échantillonnage se trouvent en des zones nettement hétérogènes. En fait, quatre points paraissent suffire : deux en amont et deux en aval, ces derniers étant situés là où l'homogénéité du mélange des eaux est parfaitement réalisée, par exemple à l'Ardoise et à Roquemaure.

- Les échantillons prélevés à point fixe (série R.1-R.8) s'avèrent aussi représentatifs que les prélèvements en continu dans l'axe du courant (série E.1-E.7). L'étude d'une section transversale du fleuve donne une image encore plus fidèle de la répartition de la contamination et est, en définitive plus intéressante que l'étude par des prélèvements longitudinaux.

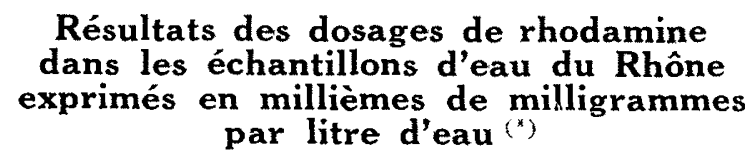

Résultats des dosages de rhodamine dans les échantillons d'eau du Rhône exprimés en millièmes de milligrammes par litre d'eau $\left.{ }^{*}\right)$

Tranche DU P.K. 211

(de la rive gauche à la rive droite) :

$\mathrm{N}^{\circ}: 1,0,1 ; 2,<0,1 ; 3,<0,1 ; 4,0,64 ; 5,1,0$; $6,2,2 ; 7,1,9 ; 8,1,9 ; 9,0,66$.

Tranche DU P.K. 214

(de la rive gauche à la rive droite) :

$\mathrm{N}^{\circ}: 1,1,2 ; 2,1,0 ; 3,0,97 ; 4,0,89 ; 5,1,0 ; 6,1,0$; $7,1,0 ; 8,1,2$.

Tranche DU P.K. 221,9

(de la rive gauche à la rive droile) :

$\mathrm{N}^{\circ}: 1,1,0 ; 2,1,1 ; 3,1,1 ; 4,<0,1 ; 5,1,2 ; 6,1,1$.

Tranche du bac D'Avignon

(de la rive gauche à la rive droite) :

$\mathrm{N}^{\circ}$ (niveau de l'eau) : $1,1,4 ; 10,0,97$.

$\mathrm{N}^{\circ}(-1 \mathrm{~m}): 2,1,1 ; 3,0,95 ; 4,0,97 ; 5,0,97$; $6,1,0 ; 7,0,97 ; 8,0,96 ; 9,0,98$.

$\mathrm{N}^{\circ}(-2 \mathrm{~m}): 1,0,98 ; 2,0,90 ; 3,0,97 ;$ 人, 0,98 ; $5,1,0 ; 6,0,97$.

$\mathrm{N}^{\circ}(-3 \mathrm{~m}): 1,1,9 ; 2,0,58 ; 3,0,98$.

Prélèvements Fixes :

Matin : R.1, 0;R.2, 0; R.3, <0.1; R.h, 0,53; $R .5,2,0 ; R .6,1,5 ; R .7,0,98 ; R .8,1,8$.

Soir : R.1,0;R.2, $0 ; R .3,3,4 ; R .1,2,1 ; R .5,2,2$; R.6, 1,$0 ; R .7,1,3 ; R .8,0,64$.

Prélèvements continus : E.1, $0 ; E .2,0,66 ; E .3$, 1,$6 ; E .4,1,1 ; E .5,1,9 ; E .6,0,98 ; E .7,1,8$.

PRÉLEVEMENTS

Dans la cuVe dE la station Roguemaure :

Heure : 11 h $30,0,96 ; 13$ h $30,0,95 ; 15$ h $30,0,67$.

$\left.{ }^{*}\right)$ Les chiffres en italique indiquent le numéro du dosage. Les chiffres en romain, le dosage de rhodaminc en $\mu \mathrm{g} /$ litre. 